\title{
A MODIFIED METHOD FOR PREDICTIVITY OF HEART RATE VARIABILITY
}

\author{
Mazhar B. Tayel ${ }^{1}$ and Eslam I AlSaba ${ }^{2}$ \\ ${ }^{1,2}$ Department of Electrical Engineering, \\ Alexandria University, Alexandria, Egypt \\ profbasyouni@gmail.com \\ eslamibrahim@myway.com
}

\begin{abstract}
Heart Rate Variability (HRV) plays an important role for reporting several cardiological and non-cardiological diseases. Also, the HRV has a prognostic value and is therefore quite important in modelling the cardiac risk. The nature of the HRV is chaotic, stochastic and it remains highly controversial. Because the HRV has utmost importance, it needs a sensitive tool to analyze the variability. In previous work, Rosenstein and Wolf had used the Lyapunov exponent as a quantitative measure for HRV detection sensitivity. However, the two methods diverge in determining the HRV sensitivity. This paper introduces a modification to both the Rosenstein and Wolf methods to overcome their drawbacks. The introduced Mazhar-Eslam algorithm increases the sensitivity to HRV detection with better accuracy.
\end{abstract}

\section{KEYWORDS}

Heart Rate Variability, Chaotic system, Lyapunov exponent, Transform domain, and Largest Lyapunov Exponent.

\section{INTRODUCTION}

Cardiovascular diseases are a growing problem in today's society. The World Health Organization (WHO) reported that these diseases make up about $30 \%$ of total global deaths. Those heart diseases have no geographic, gender or socioeconomic boundaries [3]. Therefore, early stage detection of cardiac irregularities and correct treatment are very important. This requires a good physiological understanding of cardiovascular system.

Studying the fluctuations of heart beat intervals over time reveals a lot of information called heart rate variability (HRV) analysis. A reduction of HRV has been reported in several cardiological and non-cardiological diseases. Also, HRV has a prognostic value and is therefore quite significant in modelling the cardiac risk. HRV has already proved his usefulness and is based on several articles that have reviewed the possibilities of HRV [1 - 5].

The fact that HRV is a result of both linear and nonlinear fluctuations opened new perspectives as previous research was mostly restricted to linear techniques. Some situations or interventions can change the linear content of the variability, while leaving the nonlinear fluctuations intact. In addition, the reverse can happen: interventions, which up till now have been believed to leave cardiovascular fluctuations intact based on observations with linear methods, can just as well modify the nonlinear fluctuations. This can be important in the development of new drugs or treatments for patients. This paper introduces a modification algorithm to overcome the

Jan Zizka et al. (Eds) : DBDM, CICS, CCNET, CSIP - 2015

pp. 67-77, 2015. (C) CS \& IT-CSCP 2015

DOI : $10.5121 /$ csit.2015.50706 
drawbacks arising in both Rosenstein and Wolf method using the same approach of Lyapunov exponent. That analysis the nonlinear behaviour of the HRV signals.

\section{HEART RATE VARIABILITY}

Heart Rate Variability (HRV) is a phenomenon that describes temporal variation in intervals between consecutive heartbeats in sinus rhythm. HRV refers to variations in beat-to-beat intervals corresponding to instantaneous HRs. HRV is a reliable reflection of many physiological factors modulating normal rhythm of heart. In fact, they provide a powerful means of observing interplay between sympathetic and parasympathetic nervous systems. It shows that a structure generating a signal is not only simply linear, but also involves nonlinear contributions.

Spontaneous variability of HR has been related to three major physiological originating factors: quasi-oscillatory fluctuations thought to arise in blood-pressure control, variable frequency oscillations due to thermal regulation, and respiration. Frequency selective analysis of cardiac inter-beat interval sequences allows separate contributions to be isolated. Using this method, a laboratory and field study of effects of mental work load on the cardiac interval sequence has been carried out [4].

The diagnosticity of HR is restricted by several factors like environmental stressors and physical demands that may be associated with a task. These tasks may have different physiological consequences and change in HR may depends on these factors more than mental workload. Backs (1998) [6] focused on the fact that observed HR could be caused by different underlying patterns of autonomic nervous system activity. If different information processing demands affect the heart via different modes of autonomic control, it could increase diagnosticity of HR [6]. Backs ${ }^{\text {ee }}$ study addressed the validity of the autonomic component, using data from a large study, in which many central and peripheral psycho- 8 physiological measures were collected simultaneously while performing single and dual tasks which had different physical demands. The measures collected were the residual HR, parasympathetic and sympathetic activity, respiratory sinus arrhythmia (RSA), and THM (Traube-Hering-Mayer) wave using principle component analysis (PCA), image factoring, impedance cardiogram (ZKG) and ElectroCardioGram (ECG). The sympathetic and parasympathetic systems were examined for independence. From the study, the PCA factors computed on raw ECG data provided useful information like different autonomic modes of control were found that were not evident in heart period. The objective was to verify if factors extracted using residual HR as a marker variable validly reflected cardiac sympathetic activity and if the solutions obtained from raw and baseline corrected data were in compliance with each other. This information about the underlying autonomic activity may increase the diagnosticity of HR. Figure 1 shows the example of HR variation of normal subject (control case).

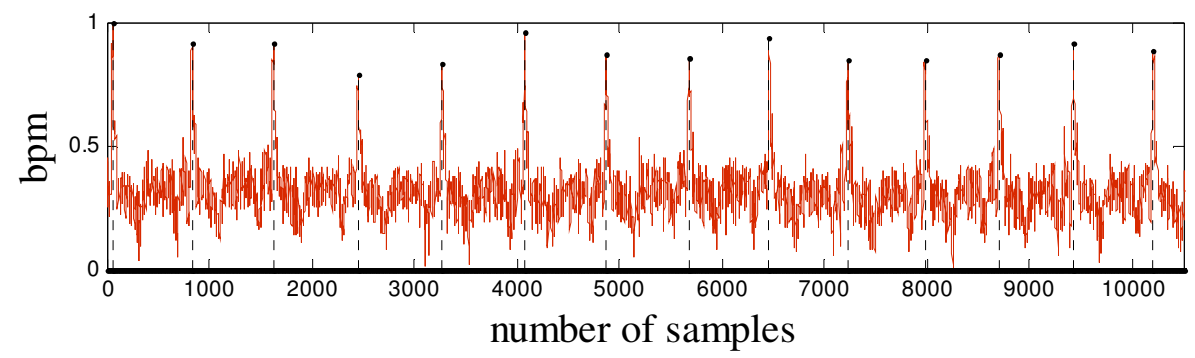

Figure 1. Heart rate variation of normal subject. 


\section{LYAPUNOV EXPONENT}

Lyapunov exponent $\Lambda$ is a quantitative measure of the sensitive dependence on the initial conditions. It defines the average rate of divergence or convergence of two neighbouring trajectories in the state-space. Consider two points in a space, $X_{0}$ and $X_{0}+\Delta x_{0}$, each of which will generate an orbit in that space using system of equations as shown in figure 2 .

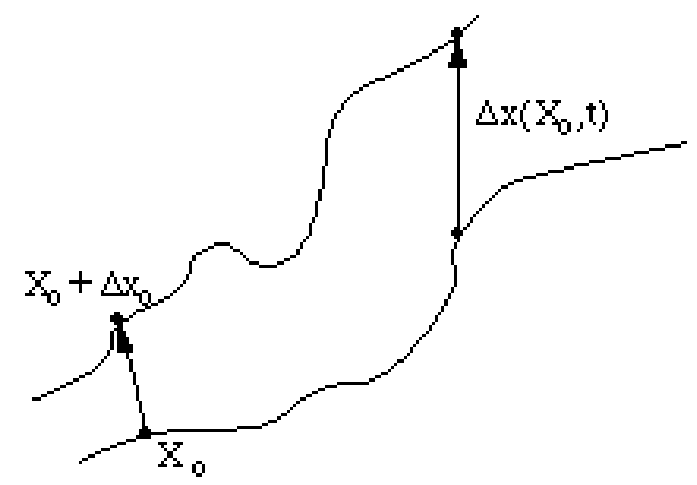

Figure 2. Calculation for two neighbouring trajectories.

These orbits are considered as parametric functions of a variable. If one of the orbits is used as a reference orbit, then the separation between the two orbits will be a function of time. Because sensitive dependence can arise only in some portions of a system, this separation is a location function of the initial value and has the form $\Delta x\left(X_{0}, t\right)$. In a system with attracting fixed points or attracting periodic points, $\Delta x\left(X_{0}, t\right)$ decreases asymptotically with time. For chaotic points, the function $\Delta x\left(X_{0}, t\right)$ will behave unpredictably. Thus, it should study the mean exponential rate of divergence of two initially closed orbits using the formula

$$
\Lambda=\lim _{\substack{t \rightarrow \infty \\\left|\Delta x_{0}\right| \rightarrow 0}} \frac{1}{t} \ln \frac{\Delta x\left(x_{0}, t\right)}{\left|\Delta x_{0}\right|}
$$

This number, called the Lyapunov exponent " $\Lambda$ ". An exponential divergence of initially nearby trajectories in state-space coupled with folding of trajectories, to ensure that the solutions will remain finite, is the general mechanism for generating deterministic randomness and unpredictability. Therefore, the existence of a positive $\Lambda$ for almost all initial conditions in a bounded dynamical system is the widely used definition of deterministic chaos. To discriminate between chaotic dynamics and periodic signals, $\Lambda \mathrm{s}$ are often used. The trajectories of chaotic signals in state-space follow typical patterns. Closely spaced trajectories converge and diverge exponentially, relative to each other. A negative exponent $(\Lambda<0)$ the orbit attracts to a stable fixed point or stable periodic orbit. Negative Lyapunov exponents are characteristic of dissipative or non-conservative systems. Such systems exhibit asymptotic stability. The more negative the exponent, the greater the stability. Super stable fixed points and super stable periodic points have a Lyapunov exponent of $\Lambda=-\infty$. This is something similar to a critically damped oscillator in that the system heads towards its equilibrium point as quickly as possible. A zero exponent $(\Lambda=0)$ the orbit is a neutral fixed point (or an eventually fixed point). A Lyapunov exponent of zero indicates that the system is in some sort of steady state mode. A physical system with this exponent is conservative. Such systems exhibit Lyapunov stability. Take the case of two identical simple harmonic oscillators with different amplitudes. Because the frequency is independent of the amplitude, a phase portrait of the two oscillators would be a pair of concentric 
circles. The orbits in this situation would maintain a constant separation. Finally, a positive exponent implies the orbits are on a chaotic attractor. Nearby points, no matter how close, will diverge to any arbitrary separation. These points are unstable [3]. The flowchart of the practical algorithm for calculating largest Lyapunov exponents is shown in figure 3.

In the following, the Largest Lyapunov Exponent algorithms are discussed.

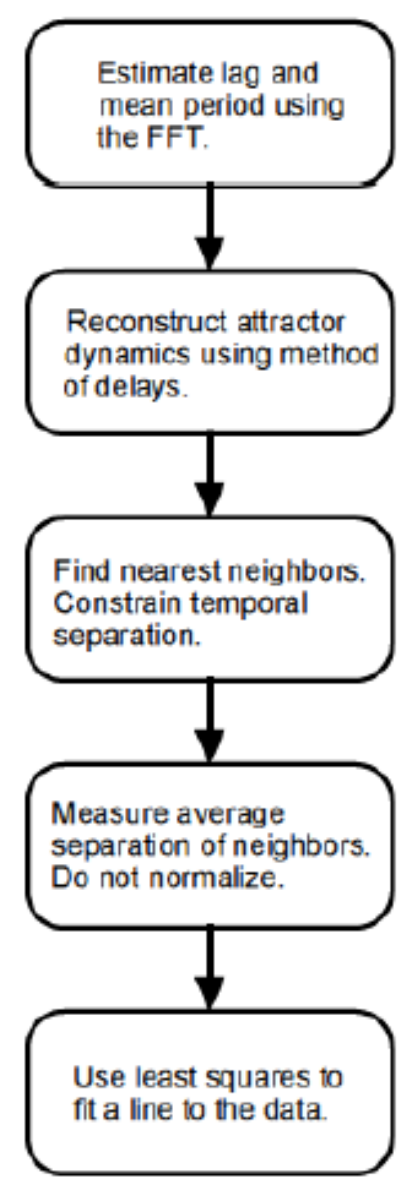

Figure 3. Flowchart of the practical algorithm for calculating largest Lyapunov exponents

\subsection{Wolf's Algorithm}

Wolf's algorithm [7] is straightforward and uses the formulas defining the system. It calculates two trajectories in the system, each initially separated by a very small interval. The first trajectory is taken as a reference, or 'fiducial' trajectory, while the second is considered 'perturbed'. Both are iterated together until their separation is large enough.

In this study, the two nearby points in a state-space ${ }^{x_{i}}$ and $x_{i}+\Delta x$, that are function of time and each of which will generate an orbit of its own in the state, the separation between the two orbits $\Delta \mathrm{x}$ will also be a function of time. This separation is also a function of the location of the initial value and has the form $\Delta x\left(x_{i}, t\right)$, where $t$ is the value of time steps forward in the trajectory. For chaotic data set, the mean exponential rate of divergence of two initially close orbits is characterized by 
$\Lambda=\lim _{K \rightarrow \infty} \frac{1}{K} \ln \frac{\left|\Delta x\left(x_{i} t\right)\right|}{|\Delta x|}$

The maximum positive $\Lambda$ is chosen as $\Lambda_{m}$.

Largest Lyapunov exponent's quantify sensitivity of the system to initial conditions and gives a measure of predictability. This value decreases for slowly varying signals like congenital heart block (CHB) and Ischemic/dilated cardiomyopathy and will be higher for the other cases as the variation of RR is more [8, 9]. The LLE is 0.505 for the HR signal shown in Figure 1.

\subsection{Rosenstein's Algorithm}

Rosenstein's algorithm [10] works on recorded time-series, where the system formulas may not be available. It begins by reconstructing an approximation of the system dynamics by embedding the time-series in a phase space where each point is a vector of the previous $\mathrm{m}$ points in time (its 'embedding dimension'), each separated by a lag of $\mathrm{j}$ time units. Although Taken's theorem [11] states that an embedding dimension of $2 \mathrm{D}+1$ is required to guarantee to capture all the dynamics of a system of order D, it is often sufficient in practice to use $m=D$. Similarly, although an effective time lag must be determined experimentally, in most cases $\mathrm{j}=1$ will suffice.

Given this embedding of the time-series, for each point founding its nearest neighbour (in the Euclidean sense) whose temporal distance is greater than the mean period of the system, corresponding to the next approximate cycle in the system's attractor. This constraint positions the neighbours as a pair of slightly separated initial conditions for different trajectories. The mean period was calculated as the reciprocal of the mean frequency of the power spectrum of the timeseries calculated in the usual manner using the Fast Fourier Transform (FFT). As shown in figure 3.

Now it can be performed a process similar to Wolf's algorithm to approximate the Largest Lyapunov Exponent (LLE). The first step of this approach involves reconstructing the attractor dynamics from the RR interval time series. After reconstructing the dynamics, the algorithm locates the nearest neighbour of each point on the trajectory. The nearest neighbour, ${ }^{\prime}{ }_{j}$, is found

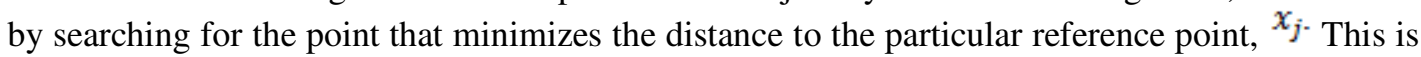
expressed as:

$$
d_{j}(0)=\min || x_{j}-x_{j} / \mid \quad \forall x_{j}
$$

where $d_{j}(0)$ is the initial distance from the ${ }^{j^{\text {th }}}$ point to its nearest neighbour and II ... II denotes the Euclidean norm. An additional constraint is imposed, namely that nearest neighbours have a temporal separation greater than the mean period of the RR interval time series. Therefore, one can consider each pair of neighbours as nearby initial conditions for different trajectories. The largest Lyapunov exponent (LLE) is then estimated as the mean rate of separation of the nearest neighbours. More concrete, it is assumed that the $j^{\text {th }}$ pair of nearest neighbours diverge approximately at a rate given by the largest Lyapunov exponent $\Lambda_{m}$ :

$$
d_{j}(j) \approx d_{j}(0) e^{\Lambda_{m}(i \cdot \Delta t)}
$$

By taking the ln of both sides of this equation: 
$\ln d_{j}(j) \approx \ln d_{j}(0)+\Lambda_{m}(i . \Delta t)$

which represents a set of approximately parallel lines (for $\mathrm{j}=1,2, \ldots, \mathrm{M}$ ), each with a slope roughly proportional to $\Lambda_{m}$ :

The natural logarithm of the divergence of the nearest neighbour to the $\mathrm{j}^{\text {th }}$ point in the phase space is presented as a function of time. The largest Lyapunov exponent is then calculated as the slope of the 'average' line, defined via a least squares fit to the 'average' line defined by:

$$
y(t)=\frac{1}{\Delta t}\left\langle\ln d_{j}(t)\right\rangle
$$

where $\{. .$.$\rangle denotes the average over all values of \mathrm{j}$. This process of averaging is the key to calculating accurate values of $\Lambda_{m}$ using smaller and noisy data sets compared to other Lyapunov algorithms. The largest Lyapunov exponent (LLE) is 0.7586 for the HR signal shown in Figure 1.

\subsection{The $\Lambda_{m}$ calculations}

The $\Lambda_{m}$ : exponents were calculated using the Wolf and Rosenstein algorithms implemented as previously recommended. For both algorithms, the first two steps were similar. An embedded point in the attractor was randomly selected, which was a delay vector with $d_{E}$ elements

$$
\left[x(t), x(t+\tau), x(t+2 \tau), \ldots, x\left(t+\left(d_{E}-1\right) \tau\right)\right]
$$

This vector generates the reference trajectory. It's nearest neighbour vector

$$
\left[x(t), x\left(t_{0}+\tau\right), x\left(t_{0}+2 \tau\right), \ldots, x\left(t_{0}+\left(d_{E}-1\right) \tau\right)\right]
$$

was then selected on another trajectory by searching for the point that minimizes the distance to the particular reference point. For the Rosenstein algorithm, it is imposed the additional constraint that the nearest neighbour has a temporal separation greater than the mean period of the time series defined as the reciprocal of the mean frequency of the power spectrum.

The two procedures then differed. For the Wolf algorithm, the divergence between the two vectors was computed and as the evolution time was higher than three sample intervals, a new neighbour vector was considered. This replacement restricted the use of trajectories that shrunk through a folding region of the attractor. The new vector was selected to minimize the length and angular separation with the evolved vector on the reference trajectory. This procedure was repeated until the reference trajectory has gone over the entire data sample and $\mathrm{k}_{1}$ was estimated as:

$$
\Lambda_{m}=\frac{1}{t_{M}-t_{0}} \sum_{k=1}^{M} \ln \frac{L^{\prime}\left(t_{k}\right)}{L\left(t_{k-1}\right)}
$$

where $L^{\prime}\left(t_{k}\right)$ and $L\left(t_{k-1}\right)$ are the distance between the vectors at the beginning and end of a replacement step, respectively, and $\mathrm{M}$ is the total number of replacement steps. Note this equation uses the natural logarithm function and not the binary logarithm function as presented by Wolf [7]. This change makes $\Lambda_{\mathrm{m}}$ exponents more comparable between the two algorithms. 
For the Rosenstein algorithm, the divergence $d(t)$ between the two vectors was computed at each time step over the data sample. Considering that $N-\left(d_{E}-1\right) \tau$ embedded points (delay vectors) composed the attractor, the above procedure was repeated for all of them and $\Lambda_{\mathrm{m}}$ were then estimated from the slope of linear fit to the curve defined by:

$y(t)=\frac{1}{\Delta t}\left\langle\ln d_{j}(t)\right\rangle$

where $\left\langle\ln d_{j}(t)\right\rangle$ represents the mean logarithmic divergence for all pairs of nearest neighbours over time. . This process of averaging is the key to calculating accurate values of $\Lambda_{m}$ using smaller and noisy data sets compared to Wolf algorithms.

\subsection{The modified Mazhar-Eslam Lyapunov Exponent}

The modified algorithm steps are same Rosenstein's algorithm steps. However, the Rosenstein's algorithm uses the Fast Fourier Transform (FFT) as shown in figure 3; the modified algorithm uses the Discrete Wavelet Transform (DWT) as shown in figure 4. That it is because DWT advantages comparing with FFT. In the next some reasons to choose DWT instead of FFT.

\subsubsection{Preference for choosing DWT instead of FFT}

Although the FFT has been studied extensively, there are still some desired properties that are not provided by FFT. This section discusses some points are lead to choose DWT instead of FFT. The first point is hardness of FFT algorithm pruning. When the number of input points or output points are small comparing to the length of the DWT, a special technique called pruning is often used [12]. However, it is often required that those non-zero input data are grouped together. FFT pruning algorithms does not work well when the few non-zero inputs are randomly located. In other words, sparse signal does not give rise to faster algorithm.

The other disadvantages of FFT are its speed and accuracy. All parts of FFT structure are one unit and they are in an equal importance. Thus, it is hard to decide which part of the FFT structure to omit when error occurring and the speed is crucial. In other words, the FFT is a single speed and single accuracy algorithm.

The other reason for not selecting FFT is that there is no built-in noise reduction capacity. Therefore, it is not useful to be used. According to the previous ,the DWT is better than FFT especially in Lyapunov exponent calculations when be used in HRV, because each small variant in HRV indicates the important data and information. Thus, all variants in HRV should be calculated.

\subsubsection{Modified calculation of Largest Lyapunov Exponent $\Lambda$}

The modified method depends on Rosenstein algorithm's strategy with replacing the FFT by DWT to estimate lag and mean period. However, the modified method use the same technique of Wolf technique for $\Lambda_{\mathrm{m}}$ calculating except the first two steps and the final step as they are taken from Rosenstein's method. The Lyapunov exponent $(\Lambda)$ measures the degree of separation between infinitesimally close trajectories in phase space. As discussed before, the Lyapunov exponent allows determining additional invariants. The modified method of LLE $(\Lambda)$ is calculated as

$$
\Lambda=\sum_{i=1}^{j} \frac{\Lambda_{i}}{j}
$$


Note that the $\Lambda_{i} \mathrm{~s}$ contain the maximum $\Lambda$ and variants $\Lambda$ s that indicate to the helpful and important data. Therefore, the modified Lyapunov is a more sensitive prediction tool. Thus, it is robust predictor for real time, in addition to its sensitivity for all time whatever the period. It is found that the modified largest Lyapunov exponent $\left(\Lambda_{\mathrm{m}}\right)$ is 0.4986 for the HR signal shown in Figure 1. Thus, it is more accurate than Wolf and Rosenstein Lyapunov exponent.

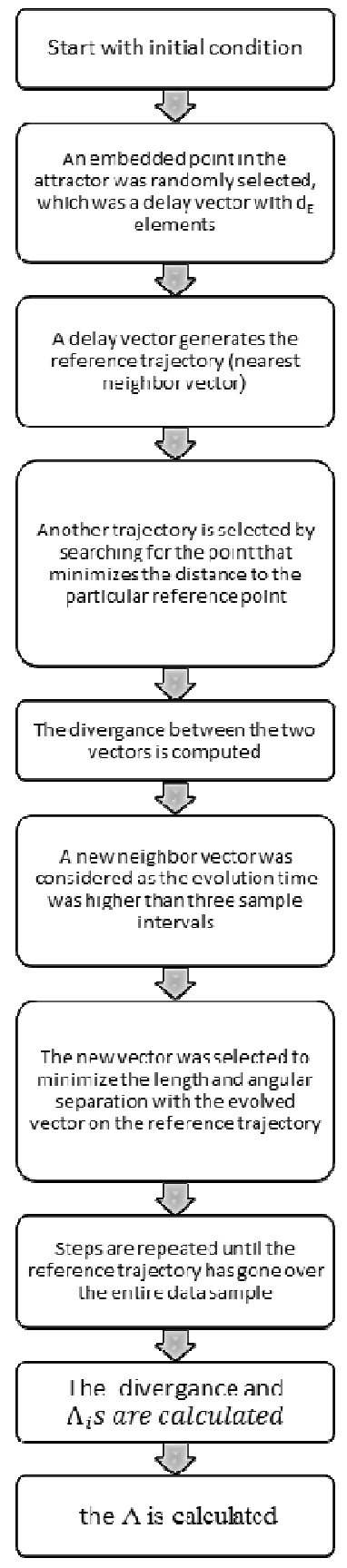

Figure 4. The flowchart of the modified algorithm.

The figure 4 shows the steps for calculating modified Mazhar-Eslam Lyapunov exponent. The modified method is the most useful and sensitive comparing to Wolf and Rosenstein methods. The table (1) discusses the different results in normal case between Modified, Wolf, and 
Rosenstein methods. The Rosenstein method is the lowest sensitive method because of its quite high error comparing to the optimum. The Wolf method takes a computational place of sensitive. However, the modified method shows more sensitivity than Wolf method as the modified error is lower than Wolf as shown in figure 5. The error for each case is calculated as

$\operatorname{error}(r)=\mid$ normal $($ optimum $)-$ case $\mid$

Thus, the accuracy for Wolf and Modified method should be calculated. The accuracy is calculated as

accurcy $=(1-r) \times 100 \%$

Figure 6 shows the accuracy of Wolf and modified method for control or normal case. It is clear that the modified Mazhar-Eslam method is more accurate than Wolf by $0.36 \%$. This result comes because the modified takes all $\Lambda$ s unlike the Wolf method as it takes only the largest. Each interval of HRV needs to be monitored and taken into account because the variant in HRV indicates to another case.

Table 1. Lyapunov result for normal case shown in figure 1

\begin{tabular}{|c|c|c|c|}
\hline Optimum & Modified method & Wolf method & Rosenstein method \\
\hline 0.5 & 0.4986 & 0.505 & 0.7586 \\
\hline Error & 0.0014 & 0.005 & 0.2586 \\
\hline
\end{tabular}

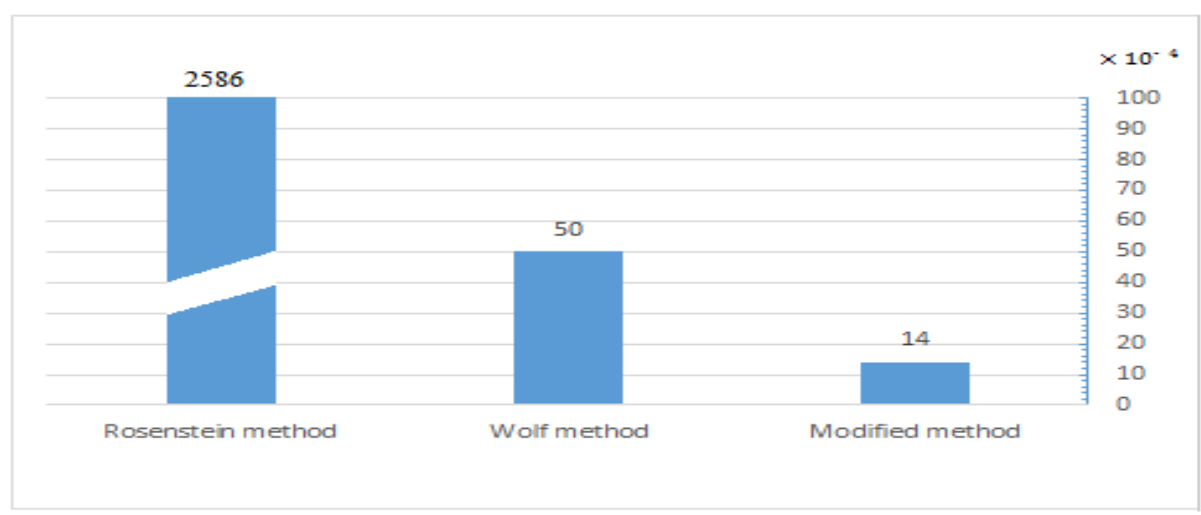

Figure 5: Methods error for the normal case.

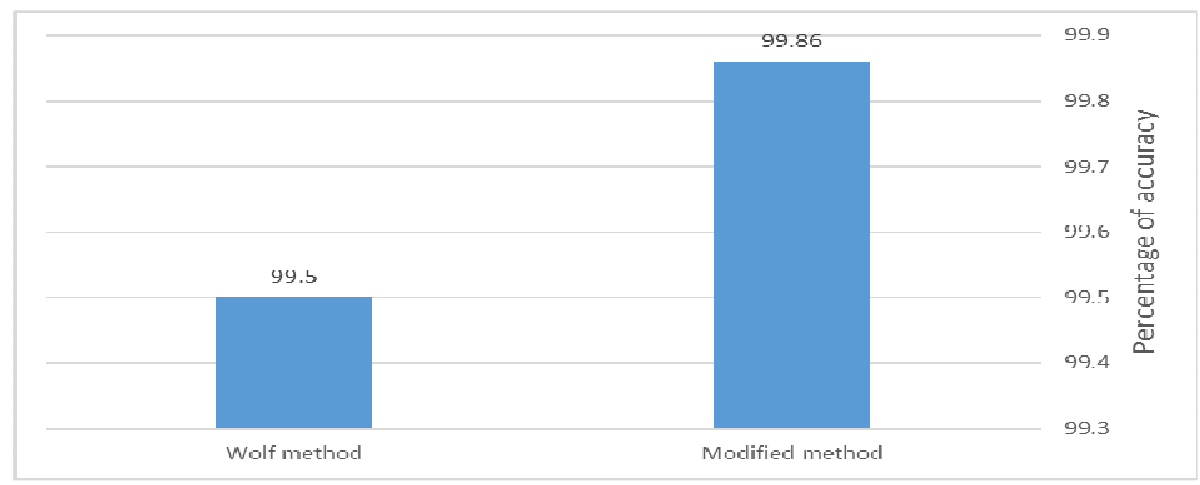

Figure 6: Accuracy percentage of Wolf and Modified method for the normal case. 


\section{CONCLUSiONS}

Heart Rate Variability (HRV) is reported in several cardiological and non-cardiological diseases. Also, it has a prognostic value and is therefore very important in modelling the cardiac risk. HRV is chaotic or stochastic remains highly controversial. In order to have utmost importance, HRV needs a sensitive tool to analyze it like Lyapunov exponent as it is a quantitative measure of sensitivity. While the $\Lambda_{\mathrm{m}}$ from both Lyapunov exponent algorithms were nearly equal for small heart rate (HR) data sets. The Rosenstein algorithm provided less sensitive $\Lambda_{\mathrm{m}}$ estimates than the Wolf algorithm to capture differences in local dynamic stability from small gait data sets. The data supported the idea that this latter outcome results from the ability and inability of the Wolf algorithm and Rosenstein algorithm, respectively, to estimate adequately $\Lambda_{\mathrm{m}}$ of attractors with an important rate of convergence as those in gait. Indeed, it was found that the Wolf algorithm makes an excellent use of the attractor divergences for estimating $\Lambda_{\mathrm{m}}$ while the Rosenstein algorithm overlooks the attractor expansion. Therefore, the Wolf algorithm appears to be more appropriate than the Rosenstein algorithm to evaluate local dynamic stability from small gait data sets like HRV. Increase in the size of data set has been shown to make the results of the Rosenstein algorithm more suitable, although other means as increasing the sample size might have a similar effect. The modified Mazhar-Esalm method combines Wolf and Rosenstein method. It takes the same strategy of Rosenstein method for initial step to calculate the lag and mean period, but it uses Discrete Wavelet Transform (DWT) instead of Fats Fourier Transform (FFT) unlike Rosenstein. After that, it completes steps of calculating $\Lambda$ s as Wolf method. The modified Mazhar-Eslam method care of all variants especially the small ones like that are in HRV. These variants may contain many important data to diagnose diseases as RR interval has many variants. Thus, the modified Mazhar-Eslam method of Lyapunov exponent $\Lambda$ takes all of $\Lambda$ s. That leads it to be robust predictor and that appear in different results between modified Mazhar-Eslam, Wolf, and Rosenstein. The Mazhar-Eslam is more accurate than Wolf and Rosenstein Lyapunov exponent. The accuracy of Wolf and modified Mazhar-Eslam method for control or normal case is more accurate than Wolf by $0.36 \%$.

\section{REFERENCES}

[1] A.E. Aubert and D. Ramaekers. Neurocardiology: the benefits of irregularity. The basics of methodology, physiology and current clinical applications. ActaCardiologica, 54(3):107-120, 1999.

[2] Task Force of the European Society of Cardiology and the North American Society of Pacing and Electrophysiology. Heart rate variability: standards of measurement, physiological interpretation and clinical use. Circulation, 93:1043-1065, 1996.

[3] U. RajendraAchrya, K. Paul Joseph, N. Kannathal, Choo Min Lim, Jasjit S. Suri. "Heart Rate Variability: a review" Med Bio EngComput (2006) 44:1031-1051

[4] P.K. Stein and R.E. Kleiger. Insights from the study of heart rate variability. Annual Review of Medicine, 50(1):249-261, 1999.

[5] Y. Gang and M. Malik. Heart rate variability analysis in general medicine. Indian Pacing and Electrophysiology Journal, 3(1):34-40, 2003.

[6] Backs, R. W. (1998). A comparison of factor analytic methods of obtaining cardiovascular autonomic components for the assessment of mental work load. [heart rate analysis]. Ergonomics. 41, 733-745.

[7] WOLF, A., SWIFT, J., SWINNEY, H., AND VASTANO, J. Determining lyapunov exponents from a time series Physica D: Nonlinear Phenomena 16, 3 (July 1985), 285-317. 
[8] Acharya UR, Kannathal N, Krishnan SM (2004) Comprehensive analysis of cardiac health using heart rate signals.

[9] Acharya UR, Kannathal N, Seng OW, Ping LY, Chua T (2004) Heart rate analysis in normal subjects of various age groups. Biomed Online J USA 3(24)

[10] ROSEnSTEIN, M. T., COLLINS, J. J., AND DE LUCA, C. J. A practical method for calculating largest lyapunov exponents from small data sets. Phys. D 65, 1-2 (1993), 117-134.

[11] Takens F 1981 Detecting strange attractors in turbulence Springer Lecture Notes in Mathematics vol 898, pp 366-81

[12] H.V. Sorensen and C.S. Burrus.Efficient computation of the DFT with only a subset of input or output points. IEEE Transactions on Signal Processing, 41(3): 1184-1200, March 1993.

\section{AUTHORS}

Mazhar B. Tayel was born in Alexandria, Egypt on Nov. 20th, 1939. He was graduated from Alexandria University Faculty of Engineering Electrical and Electronics department class 1963. He published many papers and books in electronics, biomedical, and measurements.

Prof. Dr. Mazhar Bassiouni Tayel had his B.Sc. with honor degree in 1963, and then he had his Ph.D. Electro-physics degree in 1970. He had this Prof. degree of elect. and communication and Biomedical Engineering and systems in 1980. Now he is Emeritus Professor since 1999.

From 1987 to 1991 he worked as a chairman, communication engineering section,

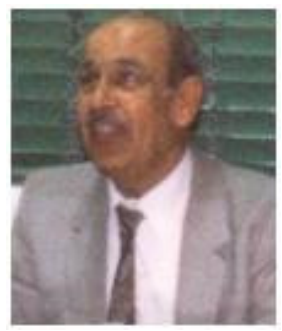
EED BAU-Lebanon and from 1991 to 1995 he worked as Chairman, Communication Engineering Section, EED Alexandria. University, Alexandria Egypt, and from 1995 to 1996 he worked as a chairman, EED, Faculty of Engineering, BAU-Lebanon, and from 1996 to 1997 he worked as the dean, Faculty of Engineering, BAU - Lebanon, and from 1999 to 2009 he worked as a senior prof., Faculty of Engineering, Alexandria. University, Alexandria Egypt, finally from 2009 to now he worked as Emeritus Professor, Faculty of Engineering, Alexandria University, Alexandria Egypt. Prof. Dr. Tayel worked as a general consultant in many companies and factories also he is Member in supreme consul of Egypt. E.Prof. Mazhar Basyouni Tayel

Eslam Ibrahim ElShorbagy AlSaba was born in Alexandria, Egypt on July. 18th, 1984. He was graduated from Arab Academy for Science Technology and Maritime Transport Faculty of Engineering and Technology Electronics and Communications Engineering department class 2007. He published many papers in signal processing. Eslam Ibrahim AlSaba had his B.Sc. with honor degree in 2007, and then he had his MSc. Electronics and Communications Engineering degree in 2010. He is a $\mathrm{PhD}$. Student in Alexandria university Faculty of Engineering Electrical department Electronics and Communications Engineering section from 2011 until now. From 2011 until now, he works as a researcher in Alexandria University. In addition, now he works as a lecturer in Al Baha International college of Science, KSA.

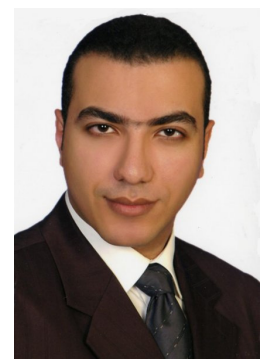

